\title{
Experimental Evaluation of the Precision of Received Signal Strength Based Visible Light Positioning
}

\author{
Willem Raes \\ ESAT-DRAMCO \\ KU Leuven \\ Ghent, Belgium \\ willem.raes@kuleuven.be
}

\author{
David Plets \\ WAVES, INTEC \\ Ghent University \\ Ghent, Belgium \\ david.plets@ugent.be
}

\author{
Lieven De Strycker \\ ESAT-DRAMCO \\ KU Leuven \\ Ghent, Belgium \\ lieven.destrycker@kuleuven.be
}

\author{
Nobby Stevens \\ ESAT-DRAMCO \\ KU Leuven \\ Ghent, Belgium \\ nobby.stevens@kuleuven.be
}

\begin{abstract}
In this work, the experimental evaluation of the distance estimation variance is executed for received signal strength based visible light positioning. It is shown that based on the signal to noise ratio at the matched filter output, an accurate determination of the precision is achieved. In order to suppress dc ambient light which contains no information regarding the distance between the LED and the receiver, matched filtering with the dc-balanced part of the transmitted signal is required. As a consequence, the theoretical lower bound for the precision can not be achieved.
\end{abstract}

\section{INTRODUCTION}

The introduction of light emitting diodes (LEDs) has changed significantly the lighting sector. Besides the known advantages such as high energy efficiency, increased life expectancy and flexible color rendering properties, LEDs have another attractive property: the light can be switched on and off at a rate that is invisible to humans, leading to a new way of wireless communication by intensity modulation of artificial light [1], known as Visible Light Communication (VLC) [2]. There are a number of drivers that motivate the research on VLC. First, there is the increasing need for a solution that alleviates the pressure on the radio frequency (RF) spectrum, due to the already large and ever increasing demand for wireless communication capacity [3]. Second, there is still the need for a cheap and accurate indoor positioning solution. The presence of a dense network of LEDs for illumination purposes is certainly an important advantage when one can deploy each LED as an anchor node. The usage of VLC for indoor positioning is called visible light positioning (VLP). A nice overview of research efforts that have been conducted the last couple of years is available in the literature [4].

In [5], the authors derive the Cramér-Rao bound (CRLB) in case of Time-of-Arrival (ToA) and received signal strength (RSS) based VLP. The CRLB is a lower bound for the variance (as the inverse of the precision). An aspect that is not investigated in detail yet is how the experimental precision of the distance estimation is related to the lower bound. It is

This work was executed within LEDsTrack, a research project bringing together academic researchers and industry partners. The LEDsTrack project was co-financed by imec and received project support from Flanders Innovation \& Entrepreneurship.

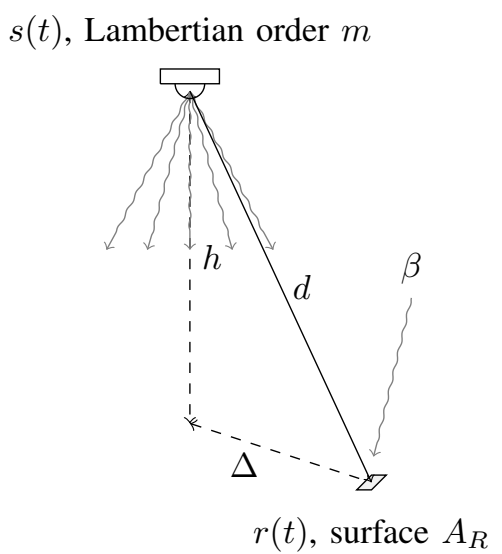

Fig. 1. The LED transmits the optical power signal $p(t)$ during $T_{s}$ and a photo diode (surface area $\left.A_{R}\right)$ with the center at $\left(x_{c}, y_{c}\right)$ receives the attenuated optical power. $\beta$ represents the dc-contribution of other ambient light sources.

clear that the lower bound is an absolute minimum, but can this lower limit be achieved in realistic circumstances? In contrast to the more theoretical elaborations on the variance [5], [6], we have constructed an experimental setup. A high number $\left(10^{4}\right)$ of distance estimations are executed at different locations, enabling the experimental evaluation of the variance. In section II, the configuration under study and related model is described. Section III focuses on the description of the experimental setup, the followed procedure to assess the precision and finally the results. In section IV, an evaluation with regard to the theoretical lower bound is performed, with the formulation of a rule of thumb to estimate the precision in case of RSS-based VLP. Finally, in section V, the main findings of this work are summarized.

\section{SySTEM DESCRIPTION}

In Fig. 1, the configuration under study is shown. A ceiling attached LED at a height $h$ above a receiver plane transmits the optical power signal $s(t)$ during a symbol time $T_{s}$, and a small fraction of this power is received by a photo diode with surface $A_{R}$, located at a planar distance $\Delta$ away from the location right below the LED. In this work, we have 
chosen $h$ to be a fixed value. This is a situation that has some important use cases, such as the planar tracking of mobile robots and forklift trucks in large warehouses. Due to the nature of signal $s(t)$, it is clear that negative values are excluded. The Euclidean spacing between the LED and receiver is $d$ (as defined by (1)). The proposed model for the received signal strength $r(t)$ is described by (2), with $R_{p}[\mathrm{~A} / \mathrm{W}]$ the responsivity of the photo diode and $n(t)[\mathrm{A}]$ a sample of an additive white Gaussian process (AWGN) with single-sided spectral density $N_{0}\left[\mathrm{~A}^{2} / \mathrm{Hz}\right]$. Relationship (2) is based on the model of [5], but we have additionally introduced $\beta$ as a dc, ambient light component (from e.g., sunlight or other light sources), matching closer with reality.

$$
\begin{gathered}
d^{2}=\Delta^{2}+h^{2} \\
r(t)=\alpha R_{p} s(t)+R_{p} \beta+n(t)
\end{gathered}
$$

The optical attenuation $\alpha$ is defined by (3), where the LED is modeled as a Lambertian radiator of order $m$. It is supposed that the LED is pointing downwards and the receiver points upwards [1], both perpendicularly to the receiver plane. Under the condition of additive white gaussian noise (AWGN), it is known that the optimal receiver is a matched filter [7], leading to the scalar $r$ as receiver output (4). Remark that due to the unknown and irrelevant contribution of ambient light sources $\beta$, we match filter the received signal $r(t)$ with $s_{a c}(t)$, where $s(t)=s_{d c}+s_{a c}(t)$. One can see that $s_{a c}(t)$ is thus the dcbalanced signal transmitted by the LED.

$$
\begin{gathered}
\alpha=\frac{(m+1)}{2 \pi} \frac{h^{m+1}}{d^{m+3}} A_{R}, \\
r=\int_{T_{s}} s_{a c}(t) r(t) \mathrm{d} t=\alpha R_{p} \mathcal{E}+n
\end{gathered}
$$

, with $\mathcal{E}$ defined as in (5)

$$
\mathcal{E}=\int_{T_{s}} s_{a c}^{2}(t) \mathrm{d} t
$$

and where $\mathbb{E}\{n\}=0$ and $\operatorname{Var}\{n\}=\mathcal{E} N_{0}$. This leads to the observation that each measured value $\hat{r}$, as an output of the matched filter, is a sample of the likelihood function $p\left(r ; \alpha R_{p} \mathcal{E}\right)[8]$

$$
p\left(r ; \alpha R_{p} \mathcal{E}\right)=\frac{1}{\sqrt{2 \pi \mathcal{E} N_{0}}} e^{-\frac{\left(r-\alpha R_{p} \mathcal{E}\right)^{2}}{2 \mathcal{E} N_{0}}} .
$$

Observation of the noise-corrupted scalar $\hat{r}$ allows estimating $\alpha$ (the estimate is $\hat{\alpha}$, see (7)), and thus $d$ (the estimate is $\hat{d}$, see (8)). When $\hat{d}$ from three or more LEDs is known, indoor positioning by means of trilateration can be achieved [9]. Considering (6), the $S N R$ can be defined as the ratio of signal power $\left(\alpha R_{p} \mathcal{E}\right)^{2}$ and noise power $\mathcal{E} N_{0}$ as in (9).

$$
\begin{gathered}
\hat{\alpha}=\frac{\hat{r}}{R_{p} \mathcal{E}} \\
\hat{d}=\left[\frac{m+1}{2 \pi} \frac{h^{m+1} A_{R}}{\hat{\alpha}}\right]^{\frac{1}{m+3}}, \\
S N R=\frac{\alpha^{2} R_{p}^{2} \mathcal{E}}{N_{0}}
\end{gathered}
$$

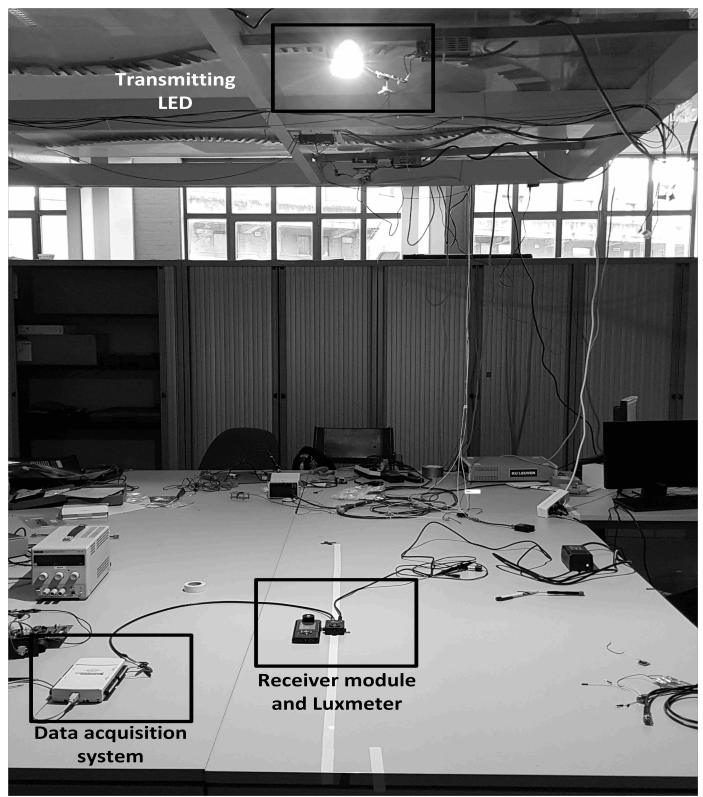

Fig. 2. Photo of the experimental setup

\section{EXPERIMENTAL SETUP AND FOLLOWED PROCEDURE}

Fig. 2 shows the setup that has been used to collect the experimental data. A commercially available LED $^{1}$ from manufacturer Bridgelux, Inc. powered with a switched mode LED driver has been configured to transmit a $300 \mathrm{~Hz}$, power switched wave with a duty cycle of $50 \%$ (equation (10), where $T_{s}=1 / 300 \mathrm{~s}$ ), with $P_{a v}$ the time averaged optical power (with a value of $4 \mathrm{~W}$ in our experiment). The LED is placed at a height $h$ of $1.381 \mathrm{~m}$ above the receiver plane. Right below the LED $(\Delta=0 \mathrm{~m})$, an average illumination of 197 lux is measured.

$$
s(t)=2 P_{a v} \operatorname{rect}\left(\frac{2 t}{T_{s}}-1\right),
$$

so that

$$
\mathcal{E}=P_{a v}^{2} T_{s}
$$

The receiver is the commercially available photo diode mod$\mathrm{ule}^{2}$ from Thorlabs, Inc. which has a built-in transimpedance amplifier with switchable gain. The effective area of the receiver is $13 \mathrm{~mm}^{2}$. Data acquisition at the output of the transimpedance amplifier is executed with the USB-62 $12^{3}$ system from National Instruments. The measurements have been conducted at 6 locations $\Delta$, starting just below the LED $(\Delta=0)$ up to $\Delta=72 \mathrm{~cm}(\Delta / h=0.521)$. At each measurement location, $10^{4}$ periods of the signal were captured and matched filtering was applied.

Since we only want to evaluate the precision of the distance estimation $\hat{d}$, a possible bias for this value was calibrated out by following procedure. At each location $\Delta$, we have $10^{4}$ samples $\hat{r}$ of the likelihood function $p\left(r ; \alpha R_{p} \mathcal{E}\right)$. Based on (4), one can see that the mean of this set $\mathbb{E}\{\hat{r}\}$ thus

\footnotetext{
${ }^{1}$ BXRE-50C3001-D-24 https://www.bridgelux.com/

${ }^{2}$ PDA36A-EC https://www.thorlabs.com/

${ }^{3}$ http://www.ni.com/nl-be/support/model.usb-6212.html
} 


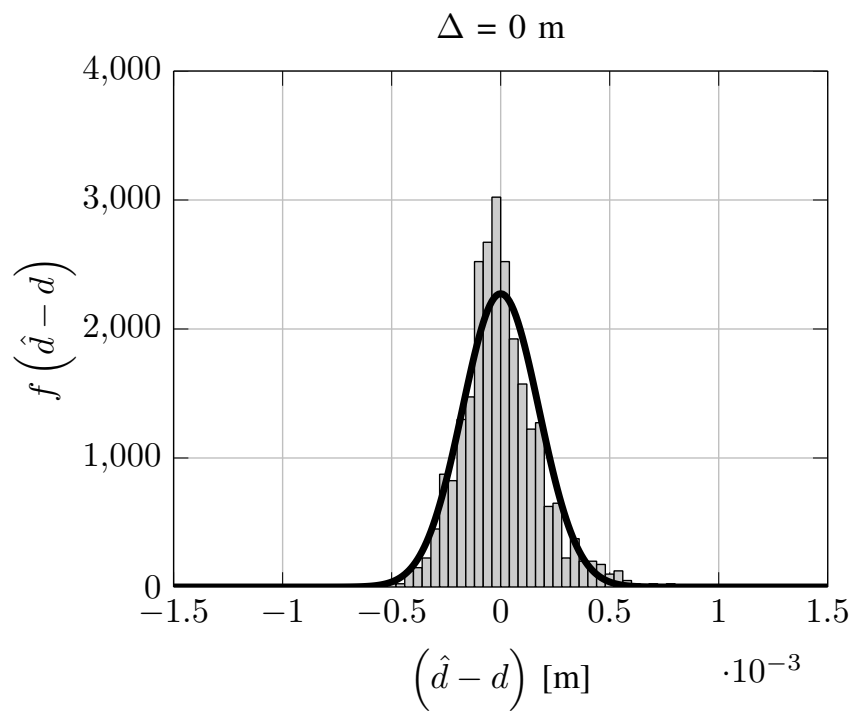

Fig. 3. Fitted gaussian probability density function $f(\hat{d}-d)$ and the measured, normalized histogram data for $\Delta=0 \mathrm{~m}$.

TABLE I

MEASUREMENT RESULTS

\begin{tabular}{|c||c|c|c|}
\hline$\Delta[\mathrm{cm}]$ & $S N R[\mathrm{~dB}]$ & $\operatorname{Var}\{\hat{d}\}\left[\mathrm{m}^{2}\right]$ & $\frac{1}{\operatorname{SNR}}\left(\frac{d}{m+3}\right)^{2}\left[\mathrm{~m}^{2}\right]$ \\
\hline \hline 0 & 65.883 & $3.07810^{-8}$ & $3.07710^{-8}$ \\
\hline 12 & 65.695 & $3.23710^{-8}$ & $3.23610^{-8}$ \\
\hline 27 & 65.335 & $3.62510^{-8}$ & $3.62310^{-8}$ \\
\hline 42 & 64.283 & $4.86010^{-8}$ & $4.85810^{-8}$ \\
\hline 57 & 62.955 & $7.07110^{-8}$ & $7.06510^{-8}$ \\
\hline 72 & 61.867 & $9.86810^{-8}$ & $9.86210^{-8}$ \\
\hline
\end{tabular}

corresponds to $\alpha R_{p} \mathcal{E}$ since $\mathbb{E}\{n\}=0$. We have manually measured the exact distance $d$ between the transmitting LED and the receiving photo diode, resulting in an exact value of $\alpha$ based on (3). We have thus that

$$
R_{p} \mathcal{E}=\frac{\mathbb{E}\{\hat{r}\}}{\alpha} .
$$

Using this relationship, we avoid the accurate assessment of $R_{p}$. Each individual measurement $\hat{r}$ of the large set $\left(10^{4}\right.$ for every location) leads to an estimation of the channel attenuation $\hat{\alpha}$ :

$$
\hat{\alpha}=\frac{\hat{r}}{R_{p} \mathcal{E}}=\alpha \frac{\hat{r}}{\mathbb{E}\{\hat{r}\}}
$$

For every value of $\hat{\alpha}$, we can, based on (8), generate an estimation of $\hat{d}$, resulting in $10^{4}$ values of $\hat{d}$, leading to an experimental assessment of the variance on $\hat{d}$. The value for the $S N R$ is easily found by evaluation of the mean and variance of the matched filter output for our set of measurement data. Since $\mathbb{E}\{n\}=0$ and $\operatorname{Var}\{n\}=\mathcal{E} N_{0}$, we have that $\mathbb{E}\{\hat{r}\}=\alpha R_{p} \mathcal{E}$, while $\operatorname{Var}\{\hat{r}\}=\mathcal{E} N_{0}$. This leads to

$$
S N R=\frac{(\mathbb{E}\{\hat{r}\})^{2}}{\operatorname{Var}\{\hat{r}\}} .
$$

Based on the described procedure, two representative histograms for the distance estimation are shown on Fig. 3

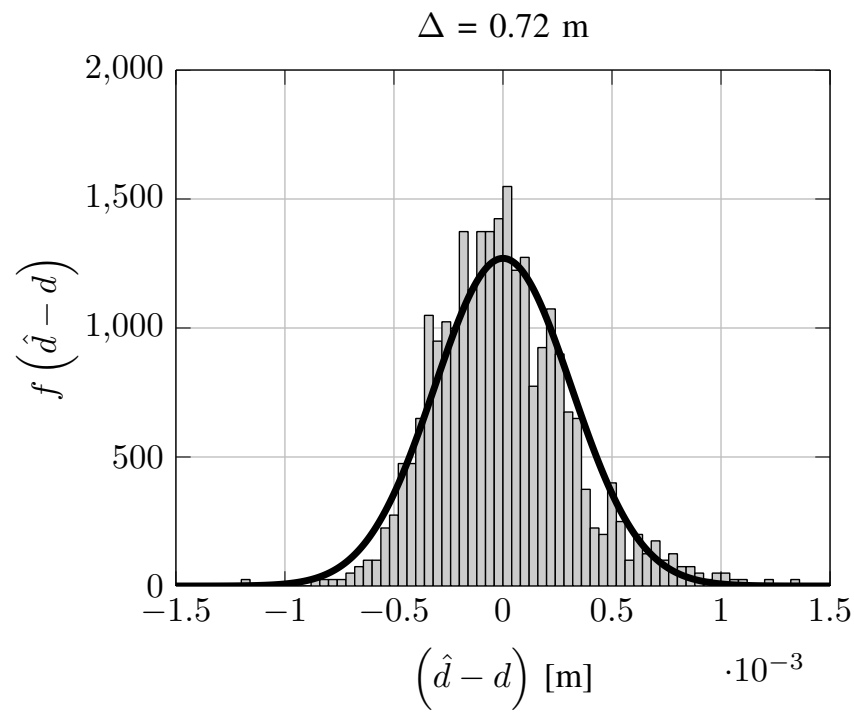

Fig. 4. Fitted gaussian probability density function $f(\hat{d}-d)$ and the measured, normalized histogram data for $\Delta=0.72 \mathrm{~m}$.

and Fig. 4 . The spread on $\hat{d}$ is clearly significantly smaller than $1 \mathrm{~mm}$ for this configuration. The measured $S N R$ and variance on the distance estimation $\operatorname{Var}\{\hat{d}\}$ can be found in respectively columns two and three of Table I, where column one represents the different transversal displacements. Corresponding to intuition, one can see that as the distance $d$ increases, the precision decreases. A legitimate question is whether these values correspond to the theoretical lower bound. This will be treated in next section.

\section{COMPARISON With THE THEORETICAL LOWER BOUND}

Keskin and Gezici [5] have demonstrated that the distance estimation variance is lower limited by the Cramér-Rao lower bound $(C R L B)$ as described by (15), (16), (17) and (18). The procedure is based on the maximization of correlation between the transmitted and received signals, resulting in the application of matched filtering with the (entire) transmitted signal $s(t)$.

$$
\operatorname{Var}\{\hat{d}\} \geq \frac{E_{1}}{E_{1} E_{2}-E_{3}} N_{0}\left(\frac{d}{\alpha R_{p}(m+3)}\right)^{2}=C R L B
$$

, where

$$
\begin{gathered}
E_{1}=\int_{T_{s}}\left(s^{\prime}(t)\right)^{2} \mathrm{~d} t, \\
E_{2}=\int_{T_{s}} s^{2}(t) \mathrm{d} t
\end{gathered}
$$

and

$$
E_{3}=\int_{T_{s}} s^{\prime}(t) s(t) \mathrm{d} t=0.5\left[s^{2}\left(T_{s}\right)-s^{2}(0)\right] .
$$

It is straightforward to define a waveform $s(t)$ such that $E_{3}$ equals zero. In our experiments, $s(t)$ fulfills this condition 
(see(10)). The variance on the distance estimation is thus reduced to (19).

$$
\operatorname{Var}\{\hat{d}\} \geqslant \frac{N_{0}}{E_{2}}\left(\frac{d}{\alpha R_{p}(m+3)}\right)^{2}
$$

Based on the distinction between the dc and non-dc part of $s(t)$, one can find the relationship between $E_{2}$ and the formerly defined $\mathcal{E}$ :

$$
E_{2}=\int_{T_{s}}\left[s_{d c}+s_{a c}(t)\right]^{2}(t) \mathrm{d} t=s_{d c}^{2} T_{s}+\mathcal{E}
$$

In our experiment though, we have to perform matched filtering with $s_{a c}(t)$, due to the presence of ambient light that has no relevance regarding the distance between the LED and the receiver. In the model by Keskin and Gezici [5], ambient light is not included. Obviously, we have that

$$
\frac{N_{0}}{E_{2}}\left(\frac{d}{\alpha R_{p}(m+3)}\right)^{2}<\frac{N_{0}}{\mathcal{E}}\left(\frac{d}{\alpha R_{p}(m+3)}\right)^{2},
$$

leading to the observation that the lower bound cannot be obtained due to the necessity of matched filtering with only the ac part of the transmitted optical signal. It is instructive though to evaluate the right hand side of (21). Considering the introduction of the $S N R$ (9), one can obtain following expression (22).

$$
\frac{N_{0}}{\mathcal{E}}\left(\frac{d}{\alpha R_{p}(m+3)}\right)^{2}=\frac{1}{S N R}\left(\frac{d}{m+3}\right)^{2}
$$

The right hand side of this relationship is easily assessed experimentally, based on (14).

$$
\frac{1}{S N R}\left(\frac{d}{m+3}\right)^{2}=\frac{\operatorname{Var}\{\hat{r}\}}{(\mathbb{E}\{\hat{r}\})^{2}}\left(\frac{d}{m+3}\right)^{2}
$$

The results of evaluating (23) for the different planar displacements $\Delta$ are shown in the fourth column of Table I. One can see that there are no significant differences compared with the histogram based precision estimation. Suppose that an LED with $m=1$ is positioned at a height $h$ of $5 \mathrm{~m}$ and that a SNR of $30 \mathrm{~dB}$ is measured right below the LED. Applying (23) would imply a standard deviation of $3.9 \mathrm{~cm}$ at that location. In the future, it is our goal to further confirm this approach for a wide range of heights, $S N R$ s and lateral displacements. Finally, we can easily evaluate how close the measured value is to the theoretical lower bound by taking the ratio of the right and left hand side of (21) when the transmitted waveform is a power switched signal with a duty cyle of $50 \%$ as described by (10):

$$
\frac{\operatorname{Var}\{\hat{\hat{d}}\}}{C R L B}=\frac{E_{2}}{\mathcal{E}}=\frac{2 P_{a v}^{2} T_{s}}{P_{a v}^{2} T_{s}}=2
$$

\section{Conclusions}

In this work, the precision of an RSS-based indoor visible light positioning system has been considered. The variance, as the inverse of the precision, is evaluated experimentally by considering a large number of measurements at different planar locations at realistic illumination levels. It is found that the obtained values correspond to a simple rule using the signal to noise ratio number at the output of the matched filter. Due to the fact that matched filtering with the dcbalanced signal is required to suppress ambient light, the lower theoretical limit can not be obtained. Furthermore, it is straightforward to determine how close the results are with regard to this lower bound.

\section{REFERENCES}

[1] T. Komine and M. Nakagawa, Fundamental analysis for visible-light communication system using LED lights, in IEEE Transactions on Consumer Electronics, vol. 50, no. 1, pp. 100-107, Feb 2004.

[2] D. Karunatilaka, F. Zafar, V. Kalavally and R. Parthiban, LED Based Indoor Visible Light Communications: State of the Art, in IEEE Communications Surveys \& Tutorials, vol. 17, no. 3, pp. 1649-1678, thirdquarter 2015.

[3] J. Lee et al., Spectrum for 5G: Global Status, Challenges, and Enabling Technologies, in IEEE Communications Magazine, vol. 56, no. 3, pp. 12-18, MARCH 2018.

[4] Do T.-H., Yoo, M. An in-Depth Survey of Visible Light Communication Based Positioning Systems, in Sensors 2016, vol. 16, no. 5.

[5] M. F. Keskin and S. Gezici, Comparative Theoretical Analysis of Distance Estimation in Visible Light Positioning Systems, in Journal of Lightwave Technology, vol. 34, no. 3, pp. 854-865, Feb.1, 12016.

[6] T. Q. Wang, Y. A. Sekercioglu, A. Neild and J. Armstrong, Position Accuracy of Time-of-Arrival Based Ranging Using Visible Light With Application in Indoor Localization Systems, in Journal of Lightwave Technology, vol. 31, no. 20, pp. 3302-3308, Oct.15, 2013.

[7] D.A. Guimaraes, Dayan Adione,Digital Transmission, Springer Berlin Heidelberg, 2009, Signals and Communication Technology.

[8] N. Stevens, D. Plets and L. De Strycker, "Monte Carlo algorithm for the evaluation of the distance estimation variance in RSS-based visible light positioning," 2017 20th International Symposium on Wireless Personal Multimedia Communications (WPMC), BALI, Indonesia, 2017, pp. 212216.

[9] A. Kupper, Location-based Services: Fundamentals and Operation, isbn 0470092319, John Wiley \& Sons, 2005.

[10] X. Zhang, J. Duan, Y. Fu, A. Shi, "Theoretical Accuracy Analysis of Indoor Visible Light Communication Positioning System Based on Received Signal Strength Indicator," Journal of Lightwave Technology, Vol. 32, No. 21, Nov 2014, pp. 4180-4186.

[11] J. M. Kahn and J. R. Barry, Wireless infrared communications, in Proceedings of the IEEE, vol. 85, no. 2, pp. 265-298, Feb 1997. 